\title{
FREE DISTRIBUTIVE P-ALGEBRAS: A NEW APPROACH ${ }^{\dagger}$ \\ by TIBOR KATRIŇÁK
}

(Received 12 December, 1996)

It is well known (Lee [13]) that the class of all distributive p-algebras $\mathbf{B}=\mathbf{B}_{\omega}$ is a variety and that the class of all subvarieties of $\mathbf{B}$ forms a chain

$$
\mathbf{B}_{-1} \subseteq \mathbf{B}_{0} \subseteq \mathbf{B}_{1} \subseteq \ldots \subseteq \mathbf{B}_{n} \subseteq \ldots \subseteq \mathbf{B}_{\omega}
$$

where $\mathbf{B}_{-1}$ is the trivial class, $\mathbf{B}_{0}$ is the class of Boolean algebras, and $\mathbf{B}_{1}$ is the class of Stone algebras.

Urquhart [14] described the finitely generated free algebras in all classes $\mathbf{B}_{n}$ for $1 \leq n \leq \omega$ (see Berman and Dwinger [4] or Köhler [12]). The free Stone algebras were studied by Balbes and Horn [3], Chen [5] and Katriñák [9],[10]. Davey and Goldberg [7] gave a characterization of the free algebras $F D p_{n}(X)$ in the classes $\mathbf{B}_{n}, 1 \leq n \leq \omega$, using the topological duality of Priestley.

In this paper we give an intrinsic algebraic characterization of $F D p_{n}(X)$ for all $1 \leq n \leq \omega$. We shall use the method of constructing the free extensions of posets in the class of distributive lattices and preserving some prescribed bounds (Dean [6]). This method has successfully been used to determine the free p-algebras $F p_{n}(X)$ in [11].

1. Preliminaries. A (distributive) $p$-algebra is an algebra $L=\left(L ; \vee, \wedge,{ }^{*}, 0,1\right)$, where $(L ; \vee, \wedge, 0,1)$ is a bounded (distributive) lattice and the unary operation ${ }^{*}$ is characterized by

$$
a \leq b^{*} \text { if and only if } a \wedge b=0
$$

In any p-algebra $L$ we can define the set of closed elements

$$
B(L)=\left\{x \in L: x=x^{* *}\right\}
$$

It is known that $\left(B(L) ;+, \wedge,{ }^{*}, 0,1\right)$ is a Boolean algebra, where

$$
a+b=\left(a^{*} \wedge b^{*}\right)^{*}
$$

As we mentioned above, the class $\mathbf{B}=\mathbf{B}_{\omega}$ of all distributive p-algebras is equational (see [2] or [8]). The subvariety $\mathbf{B}_{n}, 1 \leq n<\omega$, is defined by the following identity

$$
\left(L_{n}\right)\left(x_{1} \wedge \ldots \wedge x_{n}\right)^{*} \vee\left(x_{1}^{*} \wedge \ldots \vee x_{n}\right)^{*} \vee \ldots \vee\left(x_{1} \wedge \ldots \wedge x_{n}^{*}\right)^{*}=1
$$

(cf. [13]). Similarly as in [11], we shall work in the class of distributive lattices with the free extensions of posets preserving some bounds (see [6]): Consider a poset $(T ; \leq)$ with families $\mathcal{L}$ and $\mathcal{U}$ of finite subsets of $T$ such that

tThe support of the VEGA MS SR is gratefully acknowledged.

Glasgow Math. J. 40 (1998) 333-342. 
(i) $p \leq q$ in $T$ implies $\{p, q\} \in \mathcal{L}$ and $\{p, q\} \in \mathcal{U}$,

(ii) $S \in \mathcal{L}(S \in \mathcal{U})$ implies that there exists $\inf _{T} S\left(\sup _{T} S\right)$.

Following [6] a distributive lattice $\operatorname{FD}(T ; \mathcal{L}, \mathcal{U})$ is called a free distributive lattice generated by $T$ (a free distributive extension of $T$ ) and preserving bounds from $\mathcal{L}$ and $\mathcal{U}$, if it satisfies the following conditions:

(i) $T \subseteq \mathrm{FD}(T ; \mathcal{L}, \mathcal{U})$ and for $a, b \in T, a \leq b$ in $T$ if and only if $a \leq b$ in $\operatorname{FD}(T ; \mathcal{L}, \mathcal{U})$;

(ii) For $S \in \mathcal{L}, \inf _{T} S=\bigwedge(s: S \in S)$ and for $S \in \mathcal{U}$, $\sup _{T} S=\bigvee(s: s \in S)$;

(iii) $[T]=\mathrm{FD}(T ; \mathcal{L}, \mathcal{U})$, i.e. $T$ generates $\operatorname{FD}(T ; \mathcal{L}, \mathcal{U})$;

(iv) Let $M$ be a distributive lattice and let $\varphi: T \rightarrow M$ be an isotone mapping with the properties $\varphi\left(\sup _{T} s\right)=\bigvee(\varphi(s): s \in S)$ for every $S \in \mathcal{U}$ and $\varphi\left(\inf _{T} S\right)=\Lambda(\varphi(s): s \in S)$. Then there exists a (lattice) homomorphism $\eta: \operatorname{FD}(T ; \mathcal{L}, \mathcal{U}) \rightarrow M$ extending $\varphi$, i.e. $\eta \uparrow T=\varphi$.

2. The poset associated with a set. In order to see how to introduce this concept, we begin by observation of four facts formulated in Lemmas 1-4.

Lemma 1. Let $L$ be a distributive p-algebra. Then the following statements are equivalent:

(i) L satisfies the identity $\left(L_{n}\right), 1 \leq n<\omega$;

(ii) $\left(x_{1} \wedge \ldots \wedge x_{n+1}\right)^{*}=\left(x_{2} \wedge \ldots \wedge x_{n+1}\right)^{*} \vee \ldots \vee\left(x_{1} \wedge \ldots \wedge x_{n}\right)^{*}$;

(iii) $\left(x_{1} \vee \ldots \vee x_{n+1}\right)^{* *}=\left(x_{2} \vee \ldots \vee x_{n+1}\right)^{* *} \vee \ldots \vee\left(x_{1} \vee \ldots \vee x_{n}\right)^{* *}$;

(iv) $a_{1} \vee \ldots \vee a_{n+1}=t=a_{1}+\ldots+a_{n+1}$, whenever $a_{1}, \ldots, a_{n+1} \in B(L)$ and $a_{i}+a_{j}=t$ for any $i \neq j$.

Proof. The equivalence of (i), (ii) and (iii) can be found in Wafker [15]. Assume now (iii). Take $t=a_{1}+\ldots+a_{n+1}$ for $a_{1}, \ldots, a_{n+1} \in B(L)$ satisfying $a_{i}+a_{j}=t$ if $i \neq j$. We shall prove (iv.). For $n=1$ it follows from (iii). Assume $n \geq 2$. Let $x_{i}=a_{1} \wedge \ldots \wedge a_{i-1} \wedge a_{i+1}$ $\wedge \ldots \wedge a_{n+1}$. Then

$$
a_{i}=\left(x_{1} \vee \ldots \vee x_{i-1} \vee x_{i+1} \vee \ldots \vee x_{n+1}\right)^{* *}=x_{1}+\ldots+x_{i-1}+x_{i+1}+\ldots+x_{n+1}
$$

by distributivity and the assumption. Clearly,

$$
\left(x_{1} \vee \ldots \vee x_{n+1}\right)^{* *}=\left(a_{1} \vee \ldots \vee a_{n+1}\right)^{* *}=t
$$

Therefore,

$$
t=a_{1} \vee \ldots \vee a_{n+1}
$$

by (iii).

Conversely, suppose that (iv) is true. Take $x_{1}, \ldots, x_{n+1} \in L$ and put

$$
a_{i}=\left(x_{1} \vee \ldots \vee x_{i-1} \vee \ldots \vee x_{n+1}\right)^{* *} .
$$

It is easy to verify that

$$
\begin{aligned}
\left(x_{1}^{* *}+\ldots+x_{i-1}^{* *}+x_{i+1}^{* *}+\ldots+x_{n+1}^{* *}\right) & +\left(x_{1}^{* *}+\ldots+x_{j-1}^{* *}+x_{j+1}^{* *}+\ldots+x_{n+1}^{* *}\right) \\
& =a_{1}+a_{j}=x_{1}^{* *}+\ldots+x_{n+1}^{* *} \\
& =\left(x_{1} \vee \ldots \vee x_{n+1}\right)^{* *}=t
\end{aligned}
$$

for $i \neq j$, and this implies (iii). 
Lemma 2. Let the distributive p-algebra $L$ be generated by a subset $X$, i.e. $[X]=L$. Then the set $X^{* *}=\left\{z \in L: z=x^{* *}, x \in X\right\}$ generates $B(L)$ in the class of Boolean algebras, i.e. $\left[X^{* *}\right]_{B o o l}=B(L)$.

LeMmA 3. Let $L=\mathrm{FD} p_{n}(X)$ be a free p-algebra freely generated by $X$ in the variety $\mathbf{B}_{n}, 1 \leq n \leq \omega$. Then $B(L)=\mathrm{FB}\left(X^{* *}\right)\left(=\right.$ the free Boolean algebra freely generated by $\left.X^{* *}\right)$.

Lemma 4. Let $L$ be a distributive p-algebra generated by as subset $X$. Then $X \cup B(L)$ generates $L$ in the class of (distributive) lattices, i.e. $L=[X \cup B) L)]_{\text {Lat }}$.

Lemmas 2-4 are straightforward consequences of [11; Lemmas 2-4]. We recall that a poset associated with a set $X$ was defined in [11] as follows:

Definition 1. Let $X$ be a set. Take a disjoint copy $\bar{X}=\{\bar{x}: x \in X\}$ and construct a free Boolean algebra $\mathrm{FB}(\bar{X})$. We can assume $X \cap \mathrm{FB}(\bar{X})=\emptyset$. Now we define the poset $P(X)=(P(X) ; \leq)$ associated with $X$ as follows:

(i) $P(X)$ is bounded, i.e. $0 \leq u \leq 1$ for every $u \in P(X)$ and $0,1 \in \mathrm{FB}(\bar{X})$;

(ii) $a \leq u$ and $0 \neq a \in \operatorname{FB}(\overline{\bar{X}})$ if and only if $u \in \operatorname{FB}(\bar{X})$ and $a \leq u$ in $\operatorname{FB}(\bar{X})$;

(iii) $x \leq \bar{x}$ for every $x \in X$;

(iv) $x \leq u$ for $x \in X$ if and only if $\bar{x} \leq u$ or $x=u$.

Denote by $\mathrm{FB}(\bar{X})$ the free Boolean algebra $\left(\mathrm{FB}(\bar{X}) ;+, .{ }^{\prime}, 0,1\right)$. It remains to be said which existing glb's and lub's in $P(X)$ should be preserved.

Definition 2. Let $P(X)$ be the poset associated with the given set $X$. Set

(i) $\mathcal{L}=\mathcal{L}_{\omega}=\mathcal{L}_{1}=\ldots=\mathcal{L}_{n} \ldots$ and $A \in \mathcal{L}$ if and only if $A$ is a finite subset of $\operatorname{FB}(\bar{X})$ or $A=\{a, b\} \subseteq P(X)$ and $a \leq b$ in $P(X)$;

(ii) $\mathcal{U}=\mathcal{U}_{\omega}$, where $A \in \mathcal{U}$ if and only if $A=\{a, b\} \subseteq P(X)$ and $a \leq b$ in $P(X)$;

(iii) $A \in \mathcal{U}_{n}$ for $1 \leq n<\omega$ if and only if $A \in \bar{U}$ or $A=\left\{a_{1}, \ldots, a_{n+1}\right\}$ such that $a_{1}, \ldots, a_{n+1} \in \operatorname{FB}(\bar{X})$ and $a_{1}+\ldots+a_{n+1}=a_{i}+a_{j}$ for any $i \neq j$.

Now we shall show that the lattice $\operatorname{FD}\left(P(X) ; \mathcal{L}_{n}, \mathcal{U}_{n}\right)$ for $1 \leq n \leq \omega$ do exist. Note that $a \wedge b=a \cdot b$ for any $a, b \in \mathrm{FB}(\bar{X})$ in $\operatorname{FD}\left(P(X) ; \mathcal{L}_{n}, \mathcal{U}_{n}\right)$.

First we need a new concept from Balbes [1]. Suppose that we have a set $I$. Let $(E)$ denote a set of lattice inequalities of the form.

$$
x_{i_{1}} \wedge \ldots \wedge x_{i_{n}} \leq y_{j_{1}} \vee \ldots \vee y_{j_{m}}
$$

where $\left\{i_{1}, \ldots, i_{n}\right\},\left\{j_{1}, \ldots, j_{m}\right\} \subseteq I$. A distributive lattice $L$ is called $(E)$-free if there exists a subset $A=\left\{a_{i}\right\}_{i \in J}$ of $L$ with $I \subseteq J$ such that.

(i) $[A]=L$;

(ii) the set $\left\{a_{i}\right\}_{i \in J}$ satisfies $(E)$ i.e.

$$
a_{i_{1}} \wedge \ldots \wedge a_{i_{n}} \leq a_{j_{1}} \vee \ldots \vee a_{j_{m}}
$$

for every inequality from $(E)$;

(iii) whenever $\left\{b_{i}\right\}_{i \in J}$ is a subset of a distributive lattice $M$ such that $\left\{b_{i}\right\}_{i \in J}$ satisfies $(E)$, then there exists a homomorphism $f: L \rightarrow M$ such that $f\left(a_{i}\right)=b_{i}$ for all $i \in J$. 
THEOREM 1. Let $P(X)$ be the poset associated with a set $X$. Then $\operatorname{FD}\left(P(X) ; \mathcal{L}_{n}, \mathcal{U}_{n}\right)$ exists for every $1 \leq n \leq \omega$.

Proof. First we set $I=P(X)$. Now we define the set $\left(E_{n}\right)$ of inequalities in $\left\{x_{i}\right\}_{i \in I}$ as follows:

(a) $x_{i} \leq x_{j}$ if and only if $i \leq j$ in $P(X)$;

(b) $x_{i_{j}} \wedge \ldots \wedge x_{i_{k}} \leq x_{j}$ if and only if $\left\{i_{1}, \ldots, i_{k}\right\} \subseteq \mathrm{FB}(\bar{X})$ and $j=i_{1} \ldots i_{k}$ in $\mathrm{FB}(\bar{X})$;

(c) $x_{t} \leq x_{j_{1}} \vee \ldots \vee x_{j_{n+1}}$ if and only if $t=j_{1}+\ldots+j_{n+1}$ for $j_{1}, \ldots, j_{n+1} \in F B(\bar{X})$ and $t=j_{i}+j_{k}$ whenever $i \neq k$.

By [1; Theorem 1.9], there exists an $\left(E_{n}\right)$-free distributive lattice $H_{n}$. The properties of $H_{n}$ can be summarized in other words as follows [1; Theorem 1.8]:

(i) there exists an order preserving embedding $\varepsilon: P(X) \rightarrow H_{n}$;

(ii) $\{\varepsilon(i): i \in P(X)\}$ satisfies $\left(E_{n}\right)$;

(iii) whenever $\left\{b_{i}\right\}_{i \in I} \subseteq M$ and $M$ is a distributive lattice such that $\left\{b_{i}\right\}_{i \in I}$ satisfies $\left(E_{n}\right)$, then there exists a homomorphism $f: H_{n} \rightarrow M$ such that $f(\varepsilon(i))=b_{i}$.

Now it is easy to verify that $H_{n}=\operatorname{FD}\left(P(X 2) ; \mathcal{L}_{n}, \mathcal{U}_{n}\right)$, and the proof is complete.

REMarK 1. Following [1; Theorem 1.9] the lattice $H_{n}$ from the proof of Theorem 1 can be constructed as follows: Consider mappings

$$
s: P(X) \rightarrow \mathbf{2}=(\{0,1\})
$$

i.e. $s \in \mathbf{2}^{P(X)}$. We say that $s \in \mathbf{2}^{P(X)}$ satisfies the inequality

$$
x_{i_{j}} \wedge \ldots \wedge x_{i_{n}} \leq x_{j_{1}} \vee \ldots \vee x_{j_{m}}
$$

for $i_{1}, \ldots, i_{n}, j_{1}, \ldots, j_{m} \in P(X)$, if

$$
s\left(i_{1}\right)=\ldots=s\left(i_{n}\right)=1 \text { and } s\left(j_{1}\right)=\ldots=s\left(j_{m}\right)=0 .
$$

Let $I q\left(E_{n}\right)$ denote the set of $s \in \mathbf{2}^{P(X)}$ which satisfy the inequalities from $\left(E_{n}\right)$.

Now, define $A_{i}^{(n)}=\left\{s \in \mathbf{2}^{P(X)}: s(i)=1\right.$ and $\left.s \in \mathbf{2}^{P(X)}-I q\left(E_{n}\right)\right\}, i \in P(X)$. The sublattice of $2^{P(X)}$ generated by the set $\left\{A_{i}^{(n)}: i \in P(X)\right\}$ is a bounded distributive lattice. This lattice, [ $\left.\left.A^{(n)}: i \in P(X)\right\}\right]$, is $H_{n}$.

Our next task is to establish that $\operatorname{FD}\left(P(X) ; \mathcal{L}_{n}, \mathcal{U}_{n}\right)$ is isomorphic to $\mathrm{FD} p_{n}(X)$. For the sake of clarity we shall adapt [11; Lemmas 5-7] for the distributive case.

Lemma 5. Let $P(X)$ denote the poset associated with a set $X$. Then there exists a (unique) lattice-epimorphism

$$
\pi: H_{n}=\mathrm{FD}\left(P(X) ; \mathcal{L}_{n}, \mathcal{U}_{n}\right) \rightarrow \mathrm{FB}(\bar{X})
$$

for every $1 \leq n \leq \omega$ such that

(i) $\pi(x)=\bar{x}$ for every $x \in X$,

(ii) $\pi(a)=$ a for every $a \in \mathrm{FB}(\bar{X})$,

(iii) $u \leq \pi(u)$ for every $u \in H_{n}$,

(iv) $u \leq a$ and $a \in \mathrm{FB}(\bar{X})$ implies $\pi(u) \leq a$. 
Now, we are in position to formulate the next result.

THEOREM 2. Let $P(X)$ denote the poset associated with a set $X$. Then the free distributive $p$ algebra $\operatorname{FD} p_{n}(X)$ in the class $\mathbf{B}_{n}, 1 \leq n \leq \omega$, and the distributive lattice $H_{n}=\operatorname{FD}\left(P(X) ; \mathcal{L}_{n}, \mathcal{U}_{n}\right)$ are isomorphic as lattices. More precisely, $H_{n}$ can be considered as a p-algebra such that

(i) $u^{*}=\pi(u)^{\prime}$ for every $u \in H_{n}$,

(ii) $H_{n}=[X]$,

(iii) $B\left(H_{n}\right) \simeq\left(\mathrm{FB}(\bar{X}) ;+, .,^{\prime}, 0,1\right)$,

(iv) $H_{n} \in \mathbf{B}_{n}$, i.e. $H_{n}$ satisfies the identity $\left(L_{n}\right)$.

The proofs of Lemma 5 and Theorem 2 are essentially the same as of [11; Lemmas 5-7] and of [11; Theorem 1] (see also Lemma 1).

3. Construction of $\mathrm{FD}_{n}(X)$. Theorem 2 lacks a certain simplicity which it ought to have. Our work in this section will remedy this defect.

The following definition is crucial.

Definition 3. Let $P(X)$ denote the poset associated with a set $X$. A subset $\emptyset \neq S \subseteq P(X)$ is said to be an $n$-order-filter $(1 \leq n \leq \omega)$ if

(i) $S$ is increasing, i.e. $S=[S$,

(ii) $S \cap \mathrm{FB}(\bar{X})$ is a filter (= dual ideal) of the Boolean algebra $\mathrm{FB}(\bar{X})$,

(iii) $t=a_{1}+\ldots+a_{n+1} \in S$ and $t=a_{i}+a_{j}$ for any $i \neq j$ imply $a_{i} \in S$ for some $1 \leq i \leq n+1$, whenever $1 \leq n<\omega$ and $a_{1}, \ldots, a_{n+1} \in \mathrm{FB}(\bar{X})$.

RemarK 2. It is easy to see that a $k$-order-filter is an $n$-order-filter, whenever $k \leq n$. For the sake of simplicity we shall often say "order-filter" instead of " $\omega$-order-filter". Next we shall consider mappings $s: P(X) \rightarrow 2$. Let $\operatorname{Ker}(s)$, for $s \in \mathbf{2}^{P(X)}$, denote the set $\{i \in P(X)$ : $s(i)=1\}$.

Lemma 6. Let $P(X)$ be the poset associated with a set $X$. Then $s \in 2^{P(X)}-I q\left(E_{n}\right)^{1}$ if and only if $\operatorname{Ker}(s)$ is an $n$-order-filter $(1 \leq n \leq \omega)$.

Proof. Suppose that $s \in \mathbf{2}^{P(X)}-I q\left(E_{n}\right)$. Consider $\operatorname{Ker}(s)=S \subseteq P(X)$. Let $i \leq j$ in $P(X)$ and $i \in \operatorname{Ker}(s)$. Since $s$ does not satisfy

$$
x_{i} \leq x_{j}
$$

and $s(i)=1$, we get $s(j)=1$. Thus, $j \in \operatorname{Ker}(s)$ and $\operatorname{Ker}(s)$ is increasing. Similarly, $i_{1}, i_{2} \in S \cap \operatorname{FB}(\bar{X})$ and $j=i_{1} \cdot i_{j}$ in $\mathrm{FB}(\bar{X})$ imply $j \in S$, because $s$ does not satisfy

$$
x_{i_{1}} \wedge x_{i_{2}} \leq x_{j}
$$

Assume now that $1 \leq n<\omega$ and $t=a_{1}+\ldots+a_{n+1} \in S$ such that $a_{i}+a_{j}=t$ for any $i \neq j$ and $a_{1}, \ldots, a_{n+1} \in \mathrm{FB}(\bar{X})$. Since $s$ does not satisfy

$$
x_{t} \leq x_{a_{1}} \vee \ldots \vee x_{a_{n+1}}
$$

'The symbol - refers to the set-theoretic difference. 
for $t=a_{1}+\ldots+a_{n+1}$ and $s(t)=1$, we get $\left(a_{i}\right)=1$ for some $1 \leq i \leq n+1$. Thus, $\operatorname{Ker}(s)$ is an $n$-order-filter of $P(X)$.

The converse statement follows easily from properties of $n$-order-filters, and the proof is complete.

We now denote the set of all $n$-order-filters $S$ containing the given order-filter $M \subseteq P(X)$ by $u^{(n)}(M)$. $u^{(n)}(i)$ will simply mean $u^{(n)}(\{i\})$. Since $u^{(n)}(i)$ is a family of subsets of $P(X)$, we can write $u^{(n)}(i) \subseteq 2^{P(X)}$. Let $K_{n}(X)$ denote the (distributive) sublattice of $2^{P(X)}$ generated by $\left\{u^{(n)}(i): i \in P(X)\right\}$, i.e. $K_{n}(X)=\left[\left\{u^{(n)}(i): i \in P(X)\right\}\right]$.

Lemma 7. Let $P(X)$ denote the poset associated with a set $X$. Then there exists a lattice isomorphism $\varphi: \mathrm{FD}_{n}(X) \rightarrow K_{n}(X), 1 \leq n \leq \omega$, such that

(i) the restriction $\varphi \uparrow P(X)$ is an order-isomorphism between $P(X)$ and $\left\{u^{(n)}(i): i \in P(X)\right\}$;

(ii) the restriction $\varphi \uparrow \mathrm{FB}(X)$ is an order-isomorphism between $\mathrm{FB}(X)$ and $\left\{u^{(i)}(i): i \in \mathrm{FB}(X)\right\}$. Moreover, there exists a lattice epimorphism

$$
\tau: K_{n}(X) \rightarrow\left\{u^{(n)}(i): i \in F B(\bar{X})\right\}
$$

such that

(iii) $\tau\left(^{(n)}(x)\right)=u^{(n)}(\bar{x})$ for every $x \in X$;

(iv) $\tau\left(u^{(n)}(a)\right)=u^{(n)}(a)$ for every $a \in \mathrm{FB}(\bar{X})$;

(v) $v \leq \tau(v)$ for every $v \in K_{n}(X)$;

(vi) $u^{(n)}(i) \subseteq u^{(n)}(a)$ and $a \in \mathrm{FB}(\bar{X})$ imply $\tau\left(u^{(n)}(i)\right) \subseteq u^{(n)}(a)$ for any $i \in P(X)$.

Proof. According to Lemma 6, the mapping

$$
s \rightarrow \operatorname{Ker}(s)
$$

is an order-isomorphism between $2^{P(X)}-I q\left(E_{n}\right)$ and the set of all $n$-order-filters of $P(X)$. Therefore, by Remark $1, H_{n}$ and $K_{n}(X)$ are isomorphic as lattices. Eventually, by Theorem 2 , there exists a lattice isomorphism

$$
\varphi: \mathrm{FD} p_{n}(X) \rightarrow K_{n}(X)
$$

It is easy to verify that this isomorphism satisfies (i) and (ii). The last conditions and Lemma 5 imply (iii)-(iv).

The condition (ii) of Lemma 7 shows that $\left\{u^{(n)}(i): i \in \mathrm{FB}(\bar{X})\right\}$ is a Boolean algebra isomorphic to $\mathrm{FB}(\bar{X})$. In addition, $u^{(n)}(0)$ and $u^{(n)}(1)$ are the smallest and the greatest elements of it, respectively, and $u^{(n)}\left(a^{\prime}\right)$ is the complement of $u^{(n)}(a), a \in \operatorname{FB}(\bar{X})$.

We are now in position to state the main facts about $n$-order-filters.

Theorem 3. Let $P(X)$ denote the poset associated with a set $X$, let $1 \leq n \leq \omega$. Then the free distributive p-algebra $\mathrm{FD}_{n}(X)$ in the class $\mathbf{B}_{n}$ and the distributive lattice $\bar{K}_{n}(X)$ are isomorphic as lattices. More precisely, $K_{n}(X)$ can be considered as a p-algebra such that

(i) $v^{*}=\tau(v)^{\prime}$ for every $v \in K_{n}(X)$,

(ii) $\left\{u^{(n)}(i): i \in X\right\}$ freely generates $K_{n}(X)$ as a p-algebra in the class $\mathbf{B}_{n}$,

(iii) $B\left(K_{n}(X)\right)=\left\{u^{(n)}(i): i \in \mathrm{FB}(\bar{X})\right\} \simeq \mathrm{FB}(\bar{X})$.

The proof follows directly from Lemma 7. 
4. Finite Algebras. It is known (see [4] that a finitely generated distributive p-algebra is finite. Obviously, every finite distributive p-algebra is determined by its poset of non-zero join-irreducible elements. Theorem 3 suggests a natural way of describing this set.

Lemma 8. Let $X$ be a finite set. Assume that $i_{1}, \ldots, i_{k} \in P(X)$ and $1 \leq n \leq \omega$. Then there exists an order-filter $M \subseteq P(X)$ such that

$$
u^{(n)}\left(i_{1}\right) \cap \ldots \cap u^{(n)}\left(i_{k}\right)=u^{(n)}(M) .
$$

Conversely, for every order-filter $M \subseteq P(X)$ there exists $i_{1}, \ldots, i_{k} \in P(X)$ such that $\left(^{*}\right)$ is true.

Proof. Let $i_{1}, \ldots, i_{k} \in P(X)$ be given. Evidently,

$$
\left[i_{j}\right) \subseteq\left\{i_{1}, \ldots, i_{k}\right\} \cup\left[\tau\left(i_{1}\right) \ldots \tau\left(i_{k}\right)\right)=M \subseteq P(X)
$$

for every $j=1, \ldots, k . M$ is an order-filter and $\left({ }^{*}\right)$ can be easily verified.

On the other hand, let $M \subseteq P(X)$ be an order-filter. Clearly, $M \cap \mathrm{FB}(\bar{X})=[a)$ for some $a \in \mathrm{FB}(\bar{X})$. If $M \cap X=\emptyset$, then $u^{(n)}(a)=u^{(n)}(M)$. Let $M \cap X=\left\{i_{1}, \ldots, i_{k}\right\}$. Evidently, $\tau\left(i_{j}\right) \geq a$ for every $j=1, \ldots, k$. A simple verification shows that $\left({ }^{*}\right)$ holds true.

Recall that for $t \in \mathrm{FB}(\bar{X}) \subseteq P(X)$ and finite $X$, we can define the height function: Let $h_{B}(t)$ denote the length of a longest maximal chain in $[0, t] \cap \mathrm{FB}(\bar{X})$. It is easy to see that $h_{B}(t)$ is the number of all atoms $a \in \mathrm{FB}(\bar{X})$ such that $a \leq t$.

Lemma 9. Let $X$ be a finite set and let $M$ be an order-filter of $P(X)$. Assume that $M \cap \mathrm{FB}(\bar{X})=[t)$. Then $M$ is an $n$-order-filter for some $1 \leq n<\omega$ if and only if

$$
h_{B}(t) \leq n
$$

Proof. Suppose that $M$ is an $n$-order-filter and let $1 \leq n<\omega$ be the smallest integer with this property. We have to show that $h_{B}(t)=n$. For $t=0$ this is true. Assume that $t \neq 0$. Let $a_{1}, \ldots, a_{k}$ be all atoms of $\mathrm{FB}(\bar{X})$ with property: $a_{j} \leq t$. Consider elements $b_{1}=t \cdot a_{1}^{\prime}$, $\ldots, b_{k}=t \cdot a_{k}^{\prime} \in F B(\bar{X})$. Clearly,

$$
t=b_{1}+\ldots+b_{k} \text { and } b_{i}+b_{j}=t \text { for any } i \neq j .
$$

Since $b_{i} \leq t$ for all $i=1, \ldots, k$, we have

$$
k=h_{B}(t) \leq n
$$

We now go in the other direction. According to the choice of $n$ there exist distinct $b_{1}, \ldots, b_{n} \in \mathrm{FB}(\bar{X})$ such that

$$
t=b_{1}+\ldots+b_{n}, b_{i}+b_{j}=t
$$

for any $i \neq j$ and no $b_{i}=t$. Set

$$
a_{1}=t \cdot b_{1}^{\prime}, \ldots, a_{n}=t \cdot b_{n}^{\prime} \in \mathrm{FB}(\bar{X})
$$


$a_{1}, \ldots, a_{n}$ are distinct and $a_{i} \cdot a_{j}=0$ in $\operatorname{FB}(\bar{X})$ whenever $i \neq 0$. Since $a_{i} \neq 0$ for all $i=1, \ldots, n$, we see that $n \leq k=h_{B}(t)$. Thus $h_{B}(t)=n$.

Conversely, suppose that $h_{B}(t) \leq n$. Take $b_{1}, \ldots, b_{n+1} \in \mathrm{FB}(\bar{X})$ such that

$$
t=b_{1}+\ldots+b_{n+1} \text { and } t=b_{i}+b_{j} \text { whenever } i \neq j \text {. }
$$

We can also assume that $b_{1}, \ldots, b_{n+1}$ are distinct. Therefore, the elements

$$
a_{1}=t \cdot b_{1}^{\prime}, \ldots, a_{n+1}=t \cdot b_{n+1}^{\prime} \in \mathrm{FB}(\bar{X})
$$

are distinct and $a_{i} \cdot a_{j}=0$ in $\operatorname{FB}(\bar{X})$ whenever $i \neq j$. Since $h_{B}(t) \leq n$ we see that $a_{i}=0$ for some $1 \leq i \leq n+1$. Hence $b_{i}=t \in M$ and $M$ is an $n$-order-filter.

As our final result, we have the following theorem.

THEOREM 4. Let $P(X)$ denote the poset associated with a finite set $X$. Then $A \in K_{n}(X), 1 \leq n \leq \omega$, is a join-irreducible element in the lattice $K_{n}(X)$ if and only if $A=u^{(n)}(M)$ for some $n$-order-filter $M$.

Proof. In view of Lemma 8, every element $A \in K_{n}(X)$ can be written in the form

$$
A=u^{(n)}\left(M_{1}\right) \cup \ldots \cup u^{(n)}\left(M_{r}\right)
$$

for some order-filters $M_{1}, \ldots, M_{r} \subseteq P(X)$. Suppose now that $A \in K_{n}(X)$ is join-irreducible. Therefore, $A=u^{(n)}(M)$ for some order-filter ( $=\omega$-order-filter) $M$ of $P(X)$, and the assertion of the theorem is clear for $n=\omega$. We therefore assume that $1 \leq n<\omega$. Our aim is to show that there exists an $n$-order filter $T \subseteq P(X)$ such that

$$
A=u^{(n)}(M)=u^{(n)}(T)
$$

Let $[t)=M \cap F B(\bar{X})$. We shall proceed by induction on $h_{B}(t)$. $T=M$.

(I) Suppose that $h_{B}(t) \leq n$. It follows by Lemma 9 that $M$ is an $n$-order-filter. Hence

(II) Assume that $h_{B}(t)>n$. Moreover, if there exists an order-filter $T \subseteq P(X)$ such that $\left[t_{1}\right)=T \cap F B(\bar{X}), t>t_{1}$ and

$$
A=u^{(n)}(M)=u^{(n)}(T)
$$

then $T$ is an $n$-order-filter of $P(X)$. Without loss of generality we can assume that $M$ is no $n$ order-filter of $P(X)$. Then there exist distinct elements $b_{1}, \ldots, b_{n+1} \in \mathrm{FB}(\bar{X})$ satisfying the following conditions: $b_{i}<t$ for every $i=1, \ldots, n+1$ and

$$
t=b_{1}+\ldots+b_{n+1}=b_{i}+b_{j}, \text { whenever } i \neq j
$$

Form the following order-filters:

$$
M_{1}=Y \cup\left[b_{1}\right), \ldots, M_{n+1}=Y \cup\left[b_{n+1}\right) \text { for } Y=M \cap X .
$$

We claim that

$$
u^{(n)}(M)=u^{(n)}\left(M_{1}\right) \cup \ldots \cup u^{(n)}\left(M_{n+1}\right)
$$


Obviously, $u^{(n)}(M) \supseteq u^{(n)}\left(M_{1}\right) \cup \ldots \cup u^{(n)}\left(M_{n+1}\right)$. On the other hand, let $S \in u^{(n)}(M)$. Since $S$ is an $n$-order-filter, there is $b_{i} \in S$ for some $1 \leq i \leq n+1$. It follows from this that $S \supseteq M_{i}$. Therefore, $S \in u^{(n)}\left(M_{i}\right)$ and

$$
u^{(n)}(M)=u^{(n)}\left(M_{1}\right) \cup \ldots \cup u^{(n)}\left(M_{n+1}\right)
$$

as claimed. The hypothesis that $u^{(n)}(M)$ is join-irreducible implies that $A=u^{(n)}\left(M_{j}\right)$ for some $1 \leq j \leq n+1$. Clearly, $\left[b_{j}\right)=M_{j} \cap\left(\mathrm{FB}(\bar{X})\right.$ and $t>b_{j}$. By induction hypothesis is $M_{j}$ an $n$ order-filter of $P(X)$ and we can put $T=M_{j}$. This shows that for a join-irreducible $A \in K_{n}(X)$ there exists an $n$-order-filter $T$ of $P(X)$ with $A=u^{(n)}(T)$.

Conversely, let $A=u^{(n)}(M)$ for some $n$-order-filter $M$ of $P(X)$. Suppose that $A=C \cup D$ for some $C, D \in K_{n}(X)$. In view of Lemma 9 we can write

$$
A=u^{(n)}\left(M_{1}\right) \cup \ldots \cup u^{(n)}\left(M_{r}\right)
$$

for some order-filters $M_{1}, \ldots, M_{r}$ of $P(X)$. Since $M \in A$, there is $1 \leq j \leq r$ such that $M \in u^{(n)}\left(M_{j}\right)$. It follows that $A \subseteq u^{(n)}\left(M_{j}\right)$, and consequently, $A=u^{(n)}\left(M_{j}\right)$. This shows that $A$ is join-irreducible in $K_{n}(X)$.

REMARK 3. Another characterization of join-irreducibles from $\operatorname{FD} p_{n}(X)$ for finite $X$ is given in Urquhart [14]. A transformation which converts $n$-order-filters into elements of $\mathrm{FD} p_{n}(X)$ can be easily established: Let $M \subseteq P(X)$ be an $n$-order-filter and let $[t)=M \cap F B(\bar{X})$. Moreover, let

$$
\varphi:\left(\mathrm{FB}(\bar{X}) ;+, .,^{\prime}, 0,1\right) \rightarrow\left(\mathrm{FB}\left(X^{* *}\right) ;+, \wedge^{*}, 0,1\right)
$$

be a Boolean isomorphism given by

$$
\varphi: \bar{x} \rightarrow x^{* *} \text { for } \bar{x} \in \bar{X}
$$

Define

$$
p(M)=\bigwedge(x: x \in M \cap X) \wedge \varphi(t)
$$

Then $p(M)$ is a join-irreducible element in $\mathrm{FD} p_{n}(X)$ (in the characterization of [14]) which corresponds to $u^{(n)}(M)$.

\section{REFERENCES}

1. R. Balbes, Projective and injective distributive lattice, Pacific J. Math. 21 (1967), 405-420.

2. R. Balbes and Ph. Dwinger, Distributive lattices (Univ. Missouri Press, 1974).

3. R. Balbes and A. Horn, Stone lattices, Duke Math. J. 37 (1970), 537-546.

4. J. Berman and $\mathrm{Ph}$. Dwinger, Finitely generated pseudocomplemented distributive lattices, $J$. Austral. Math. Soc. 19 (1975), 238-246.

5. C. C. Chen, Free Stone extensions of distributive lattice, Nanta Math. 2 (1965), 1-15.

6. R. A. Dean, Free lattices generated by partially ordered sets and preserving bounds, Canad. $J$. Math. 16 (1964), 136-148. 
7. B. A. Davey and M. S. Goldberg, The free p-algebra generated by a distributive lattice, Algebra Univ. 11 (1980), 90-100.

8. G. Grätzer, General lattice theory (Akademie-Verlag, 1978).

9. T. Katriňák, Die freien Stoneschen Verbände und ihre Tripelcharakterisierung, Acta Math. Sci. Hungar. 23 (1970), 315-326.

10. T. Katriňák, A new description of the free Stone algebra, Algebra Univ. 5 (1975), 179-189.

11. T. Katriňák, Free p-algebras, Algebra Univ. 15 (1982), 176-186.

12. P. Köhler, The triple method and free distributive pseudocomplemented lattices, Algebra Univ. 8 (1978), 139-150.

13. K. B. Lee, Equational classes of distributive pseudocomplemented lattices, Canad. J. Math. 22 (1970), 881-891.

14. A Urquhart, Free distributive pseudo-complemented lattices, Algebra Univ. 3 (1973), 13-15.

15. P. Wafker, Generalization of a theorem on distributive pseudocomplemented lattices, (preprint).

KATČ MFF UK

MLYNSKÁ DOLINA

84215 BRATISLAVA

SLOVAKIA 